NASA Technical Memorandum 107268

\title{
Dimension Determination of Precursive Stall Events in a Single Stage High Speed Compressor
}

Michelle M. Bright

Lewis Research Center

Cleveland, Ohio

Helen K. Qammar and Tom T. Hartley

University of Akron

Akron, Ohio

Prepared for the

Third Technical Conference on Nonlinear Dynamics and Full Spectrum Processing sponsored by the Office of Naval Research

Mystic, Connecticut, July 10-14, 1995

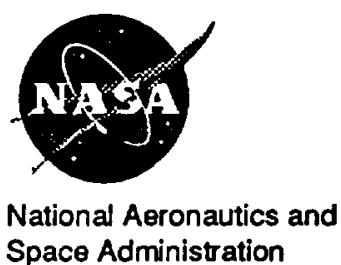

Space Administration 


\title{
Dimension Determination of Precursive Stall Events in a Single Stage High Speed Compressor
}

\author{
Michelle M. Bright \\ NASA Lewis Research Center \\ Adranced Comtrols Tedmology and System Dynamics Branches \\ Clevelesind, OH 7413.5 \\ Helen K. Qammar and Tom T. Hartley \\ Departments of Chemical and Electrical Engineering \\ The University of Akron \\ Akron, Ohio 44325
}

\begin{abstract}
This paper presents a study of the dynamics for a single-stage, axial-flow, high speed compressor core, specifically, the NASA Lewis rotor stage 37. Due to the overall blading design for this advanced core compressor, each stage has considerable tip loading and higher speed than most compressor designs, thus, the compressor operates closer to the stall margin. The onset of rotating stall is explained as bifurcations in the dynamics of axial compressors. Data taken from the compressor during a rotating stall event is analyzed. Through the use of a box-assisted correlation dimension methodology. the altractor dimension is determined during the bifurcations leading to rotating stall. The intent of this study is to examine the behavior of precursive stall events so as to predict the entrance into rotating stall. This information may provide a better means to identify, avoid or control the undesireable event of rotating stall formation in high speed compressor cores.
\end{abstract}

\section{MOTIVATION}

In studying the dynamics of turbomachinery, specifically, axial-now compressor behavior, several approaches have been developed to determine when the compressor is entering a rotating stall event. Rotating stall, as shown in Figure 1 , is the development of a circumferentially non-uniform flow across the compressor face. Surge, on the other hand, is an axially oscillating flow event through the compressor. The rotating stall event is consequentially severe hecause 
stall can cause severe unsteady loading on the rotor and stator blades. Rotating stall causes a large drop in pressure rise delivered in the engine where an engine restart is often needed to clear the stall event. Rotating stall may also lead to surge or "flame out" of the engine. Obviously, this is a good behavior to avoid in axial-flow compressors.

ROTATING STALL

SURGE

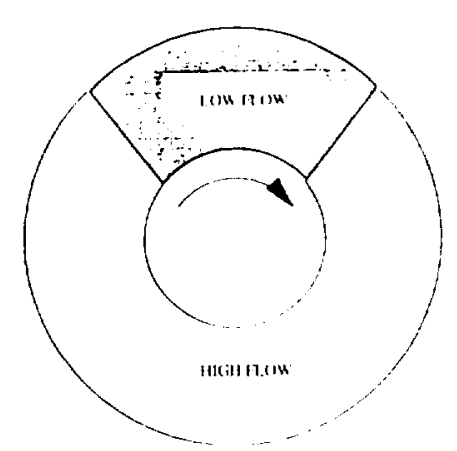

CIRCUMFERENTIALIY

NONUNIFORM FIOW

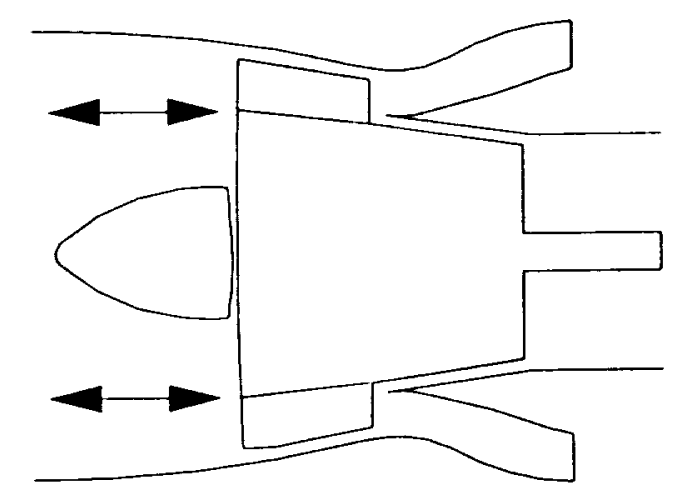

AXIALLY OSCILI.ATING FI.OW

Figure 1. Rotating Stall and Surge Events in an Axial Flow Compressor

One approach for determining prestall behavior of the compressor exists based on the assumption that rotating stall is primarily a linear event and can be approximated by the first 3 spatial harmonics of the Fourier transform coefficients. "Measurement of prestall perturbations are made by acquiring time traces from a circular array of sensors. These signals are decomposed into spatial harmonics, a spectrum of spatial harmonics is used for identification of the evolution of $2-\mathrm{D}$ rotating stall.'”[1]

Another approach uses the change in energy of the power spectral density traces of pressure data prior to stall. "In steady state behavior of the compressor, these power spectral density curves remain symmetric. However, during the evolution of stall cells these power spectral density curves change shape. This method identifies traveling wave energy in the compressor which may lead to the formation of stall cells." [2] This metamorphosis of the power spectral density curves is another precursive event for identifying rotating stall. 
The approach used in this paper attempts to identify the stall precursive events as correlation dimension changes in the pressure data from a single sensor position. This paper will describe the compressor used for the experiment, equations of motion which capture some of the relevant 1-D compressor dynamics, the data acquisition and experimental setup, the correlation dimension technique used for analysis, and a summary of observations.

\section{DESCRIPTION OF COMPRESSOR STAGE AND EXPERIMENT}

The NASA rotor 37 axial-1low, high-speed compressor stage for advanced airbreathing engines is an inlet stage compressor designed as part of an eight-stage core compressor having a pressure ratio of 20:1. The compressor design has a constant meanline diameter, with an inlet hub-tip ratio of 0.7 and an inlet rotor-tip speed of 455 meters per second. The loading per stage is considerably high for a research test compressor. Additional details on the blading, geometry, and testing of this compressor can be found in references [ 3 and 4 ].

The rotor 37 compressor was instrumented with 8 circumferentially placed pressure transducers within 1 chord length upstream of the rotor. These wall static pressure transducers measured the pressure fluctuations during the transition into stall. The rotor was stalled by closing the throttle area in a linear manner. Data was acquired for 15 seconds prior to a full stall event at a sampling rate of 2 kilohertz. The rotor was operating at $100 \%$ speed, $17581 \mathrm{rpm}$, for the stall runs. The test facility for this compressor is shown in Figure 2 which details the flow path through the inlet, compressor, plenum and throttle. Figure 3 shows the 8 circumferentially placed transducers around the wall of the compressor. 


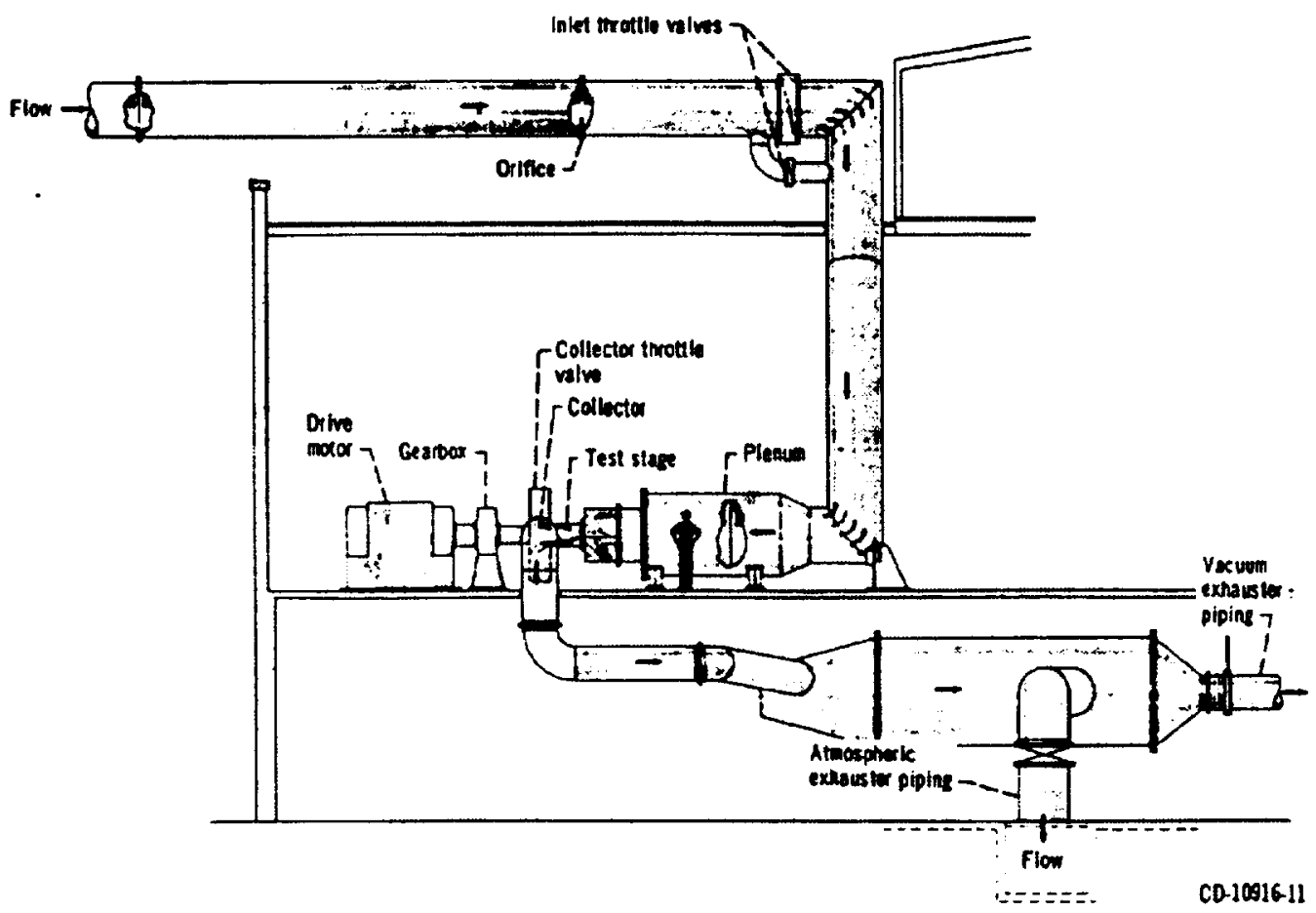

FIGURE 2. Compressor Facility Drawing of Flow Path

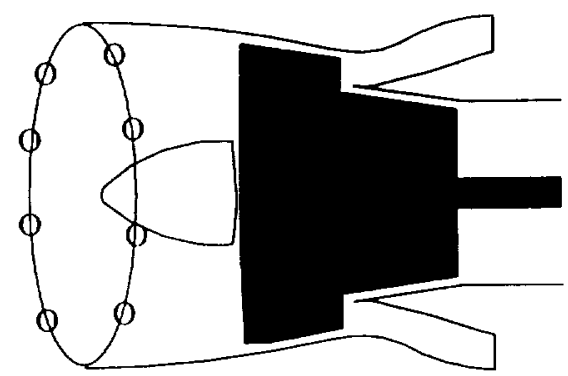

FIGURE 3. Circumferentially Placed Pressure Transducers

\section{EQUATIONS OF MOTION}

An approximate set of equations that describe surge and rotating stall transient formation are as follows from reference [5]:

$$
\begin{array}{ll}
\text { 1-D momentum: } & d \phi / d t=-\psi+\psi(\phi)-3 \phi R \\
\text { 1-D continuity: } & d \psi / d t=1 / \beta^{2}\left[\phi-\phi_{r}(\psi)\right] \\
\text { Stall cell growth: } & d R / d t=\sigma R\left(1-\phi^{2}-R\right)
\end{array}
$$


where $\phi=$ compressor mass flow, $\psi=$ system pressure rise, $\mathrm{R}=$ (stall cell amplitude $)^{2}$.

From these equations one can estimate the rate of stall cell growth for a particular compressor geometry. With this estimated rate of stall cell growth, the data collection for the experiment was planned to capture the initial stall formation with the wall static pressure transducers. Additionally, initial estimates for the embedding dimension and time delay parameters for correlation dimension determination were based on the use of this physical model.

\section{EXPERIMENTAL DATA} in Figure 4.

Data gathered from 1 of the circumferential pressure transducers is shown

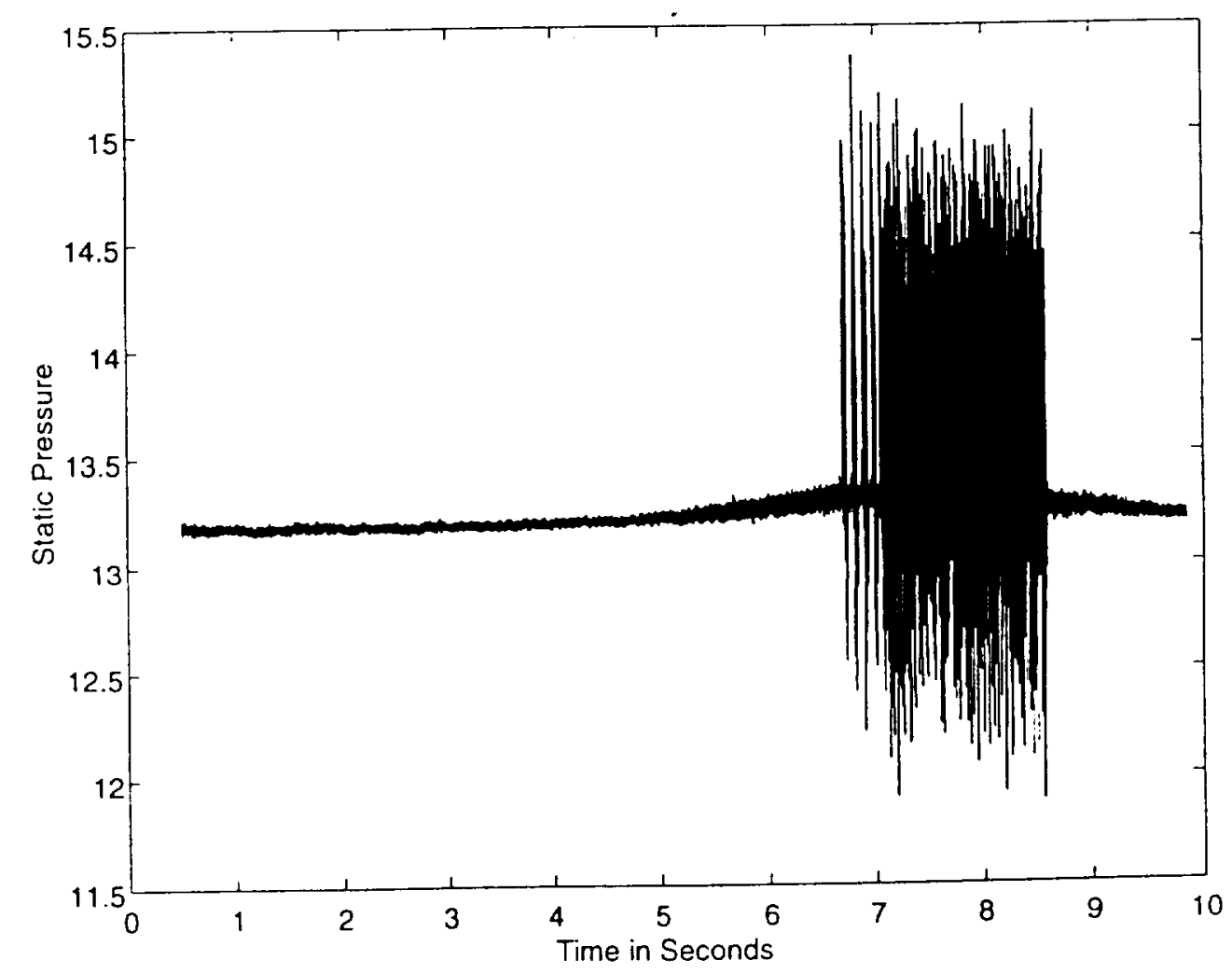

FIGURE 4. Pressure data

A phase plane portrait of the pressure data is reconstructed using 5000 data points. To reconstruct the attractor, an independent time series of data was generated 
using the following time delay embedding method[6]:

$$
v(t)=(x(t), x(t+T), \ldots, x(t+(m-1) T)
$$

where $\mathrm{v}(\mathrm{t})$ is the independent time series, $\mathrm{m}$ is the embedding dimension, and $\mathrm{T}$ is the reconstruction time delay. For this pressure data, the values of $m=4$ and $\mathrm{T}=240$ were used for the reconstruction

\section{DIMENSION DETERMINATION}

Verification of the dimension variation of this compressor is provided through a chaos package software routine. A box-assisted correlation dimension algorithm $[7,8]$ is used to determine the correlation dimension of the measured stall data.

For the correlation dimension method, 16000 pressure data samples are used prior to the onset of stall, of which the first 1000 points are thrown out as transient. Using a 5000 data point sliding window, the window is moved every 300 samples, thus creating 28 slices of 5000 points each of pre-stall data. Using 5000 data points allows for accurate prediction of correlation dimension up to approximately dimension 8 , according to the following equation from Eckmann and Ruelle[9] which states:

Maximum Accurate Dimension $<2 \log$ (number of data points).

With this limit in mind, the correlation dimension, (or correlation exponent), was calculated using a Grassberger-Procaccia correlation integral [10] for each of the 28 slices before stall. This correlation exponent is closely related to the fractal dimension and gives a lower bound to the fractal dimension. The GrassbergerProcaccia algorithm using Theiler's method[7,8] considers the spatial correlation between pairs of points on a reconstructed attractor, and is measured with the following correlation integral:

$$
C(N, r)=\frac{2}{N(N-1)} \sum_{i, j=1}^{N} H(r-\|X i-X j\|)
$$

where $H(x)$ is the Heaviside step function. The summation counts the number of pairs $(\mathrm{Xi}, \mathrm{Xj})$ for which this distance is less than $r$, where $r$ is the box size. Then

$C(N, r)$ scales like a power of $v$ so that: 


$$
\mathrm{C}(\mathrm{N}, \mathrm{r})=r^{\prime \prime}
$$

where $v$ is the correlation dimension and is the slope of the $\log -\log$ plot of $C(N, r)$ vs. $r$.

Figure 5 shows the correlation dimension integral plots for the measured pressure data. It is shown that the correlation dimension for the first 20 slices of data remains constant. However, during the $21 \mathrm{st}$ slice of data, the correlation dimension begins to increase. This corresponds to a bifurcation occurring in the pressure data and subsequently to a stall precursive event as detected from the power spectral density curves shown in Figure 6. As the slices of data move further into the onset of stall the correlation dimension continues to increase. This is shown in Figure 7.

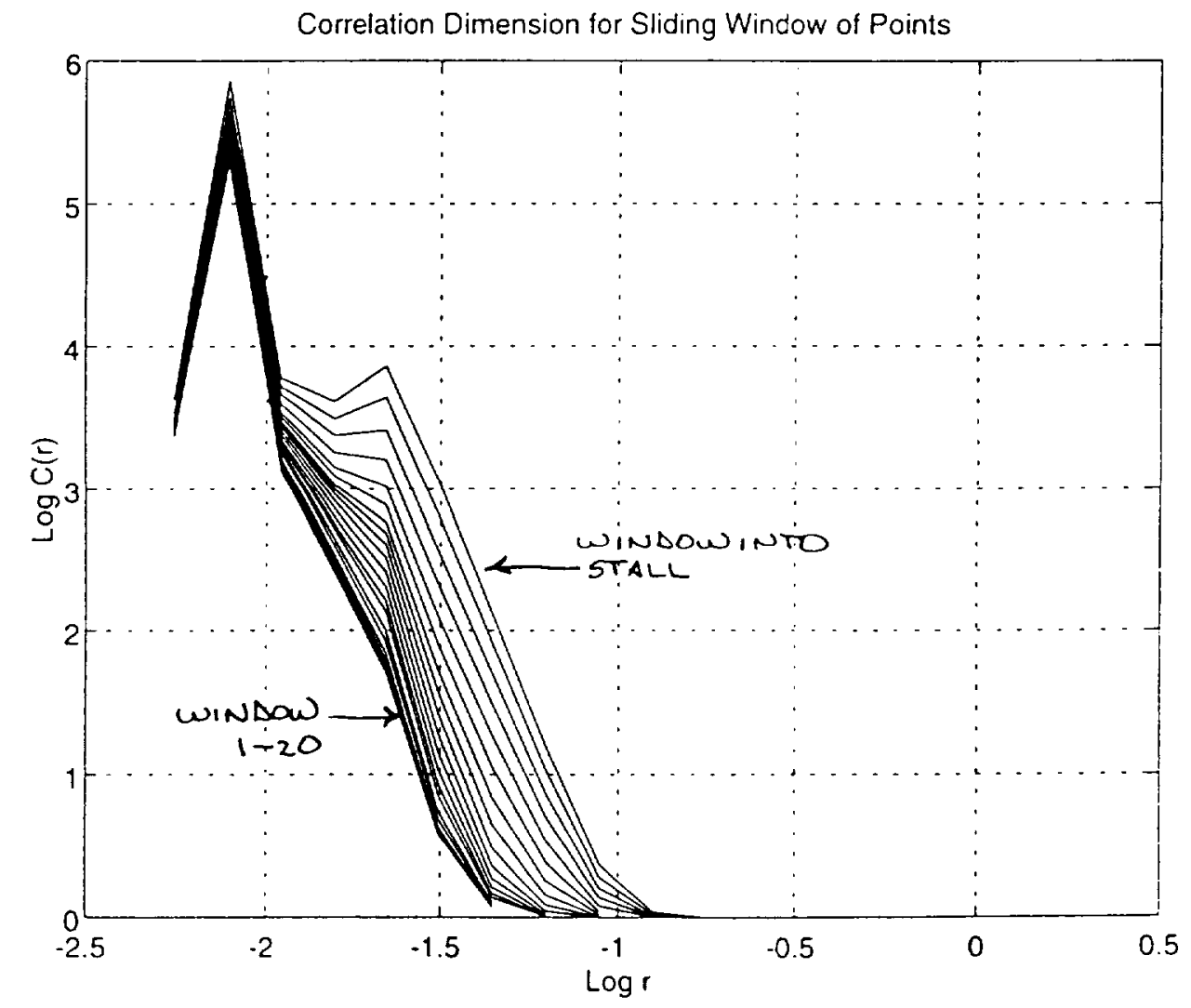

FI(:URE 5. Correlation Dimension Changes at 20 hin Window and into Stall 


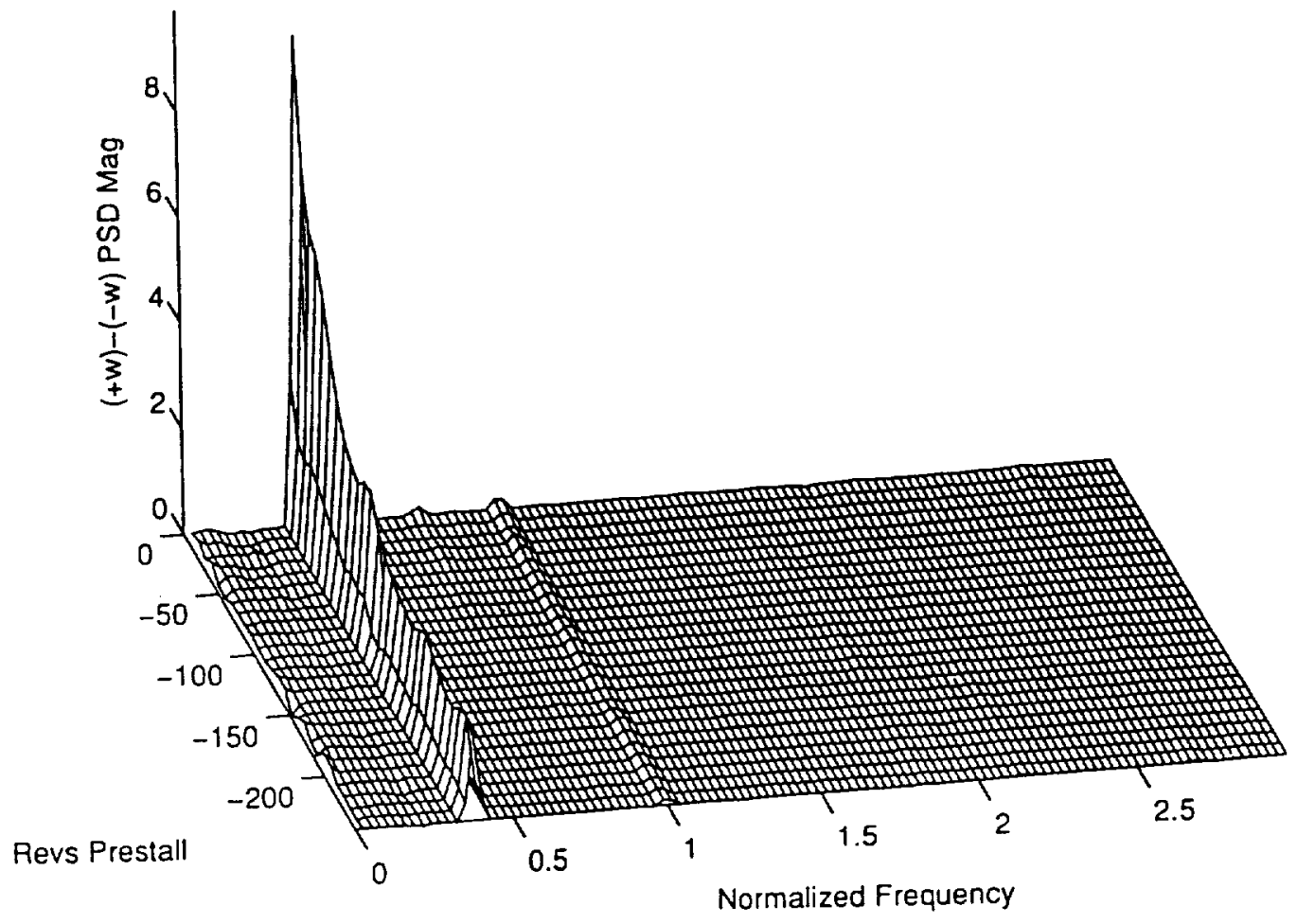

FIGURE 6. Power Spectral Density Plot Showing Stall Formation at 200 Revs Before Stall

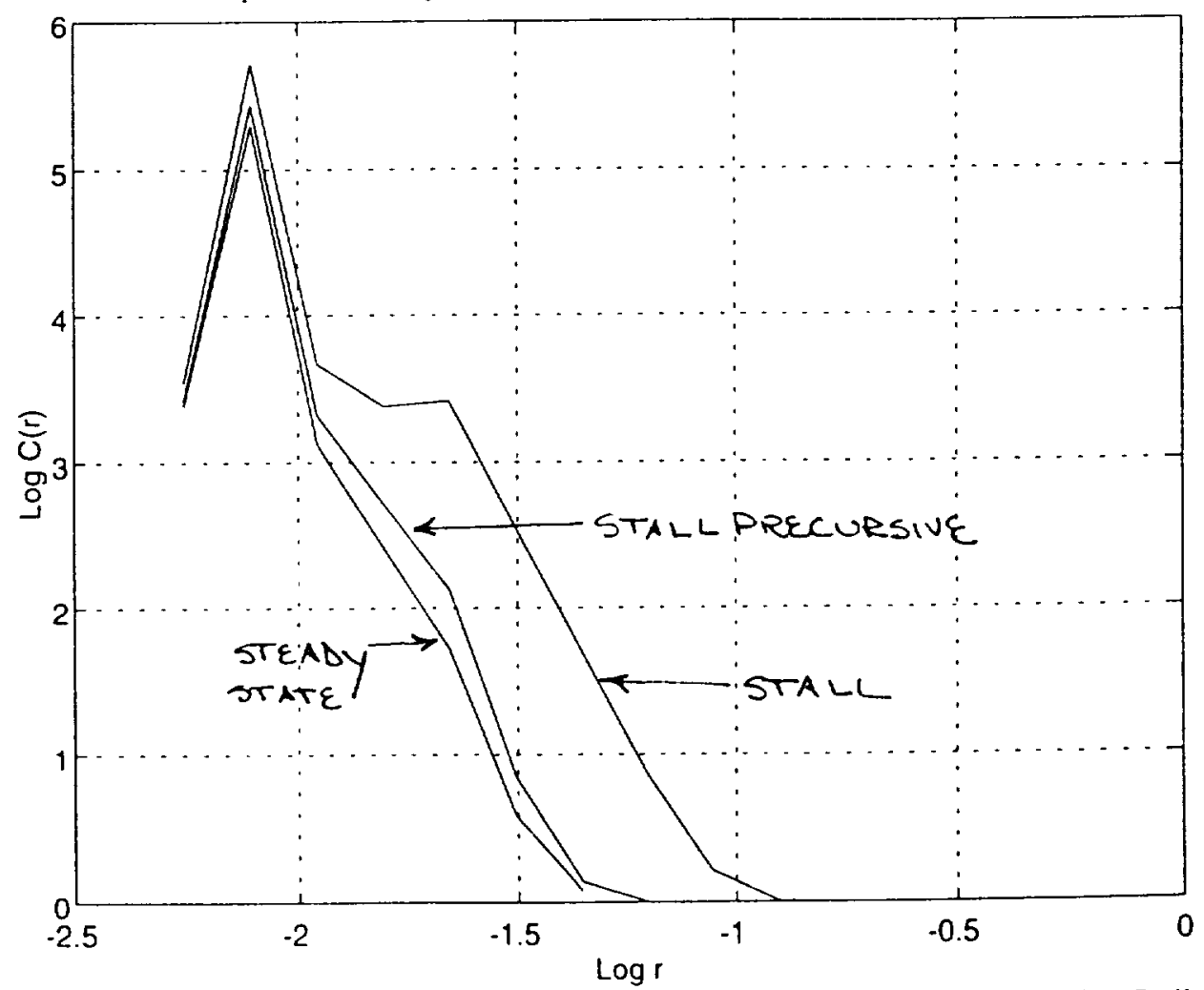

FIGURE 7. Correlation Dimension in Steady State, Prior to Stall, and In Rotating Stall 


\section{CONCLUSIONS}

From this study it is shown that pressure data acquired from a single stage, high speed compressor bifurcates during the onset of rotating stall. During these bifurcations the power spectral density and the correlation dimension is analyzed from a single pressure sensor. It is shown that the correlation dimension increases during the stall precursive events leading to rotating stall. Changes in the correlation dimension integral match the results from analyzing the power spectral density curves for this pressure data. This indicates both the correlation dimension and the power spectral density curves begin to move several hundred rotor revolutions prior to a full blown rotating stall event. This shows that correlation dimension determination may help identify rotating stall precursors in high speed axial compressors. Further work is needed to compare these results with other existing methodologies for compressor stall identification and to accurately determine if rotating stall is a chaotic event.

\section{REFERENCES}

[1] J.D. Paduano, "Active Control of Rotating Stall in Axial Compressors," MIT PhD Dissertation, March 1992.

121 Olivier Etchevers, "Evaluation of Rotating Stall Warning Schemes for Axial Compressors", MIT Masters Thesis, September 1992.

131 Lonnie Reid and Royce Moore, "Design and Overall Performance of Four Highly Loaded, High-Speed Inlet Stages for an Advanced High-Pressure-Ratio Core Compressor", NASA Technical Paper 1337, 1978.

[4] Royce Moore and Lonnie Reid, "Performance of Single-Stage Axial-Flow Transonic Compressor with Rotor and Stator Aspect Ratios of 1.19 and 1.26, Respectively, and with Design Pressure Ratio of 2.05”, NASA Technical Paper 1659, April 1980.

15] E.M. Greitzer, "Overview of Flow Instabilities in Aeroengines and Methods for Stability Enhancement", Ohio Acrospace Institute Lectures, July 1992.

[6] N.H. Packard, et. al., "Geometry from a Time Series", Physical Review Letters, Volume 45. pp. $712-716,1980$.

171 James Theiler, "Efficient Algorithm for Estimating the Correlation Dimension from a Set of Discrete Points", Physical Review A, Volume 36, No. 9, p. 4456-4462.

[8] Jammes Theiler, "Spurious Dimension from Correlation Algorithms Applied to Limited TimeSeries Dala", Physical Review A. Volume 34, No. 3, p.2427-2432. 
19] J.-P. Eckmann and D. Ruelle, "Fundamental Limitations for Estimating Dimensions and Lyapunov Exponents in Dynamical Systems", Physica D, Volume 56, 1992, pp. 185-7.

[10] Peter Grassberger and Itamar Procaccia, "Characterization of Strange Attractors", Physical Review Letters, Volume 50, No. 5, p. 346-349. 



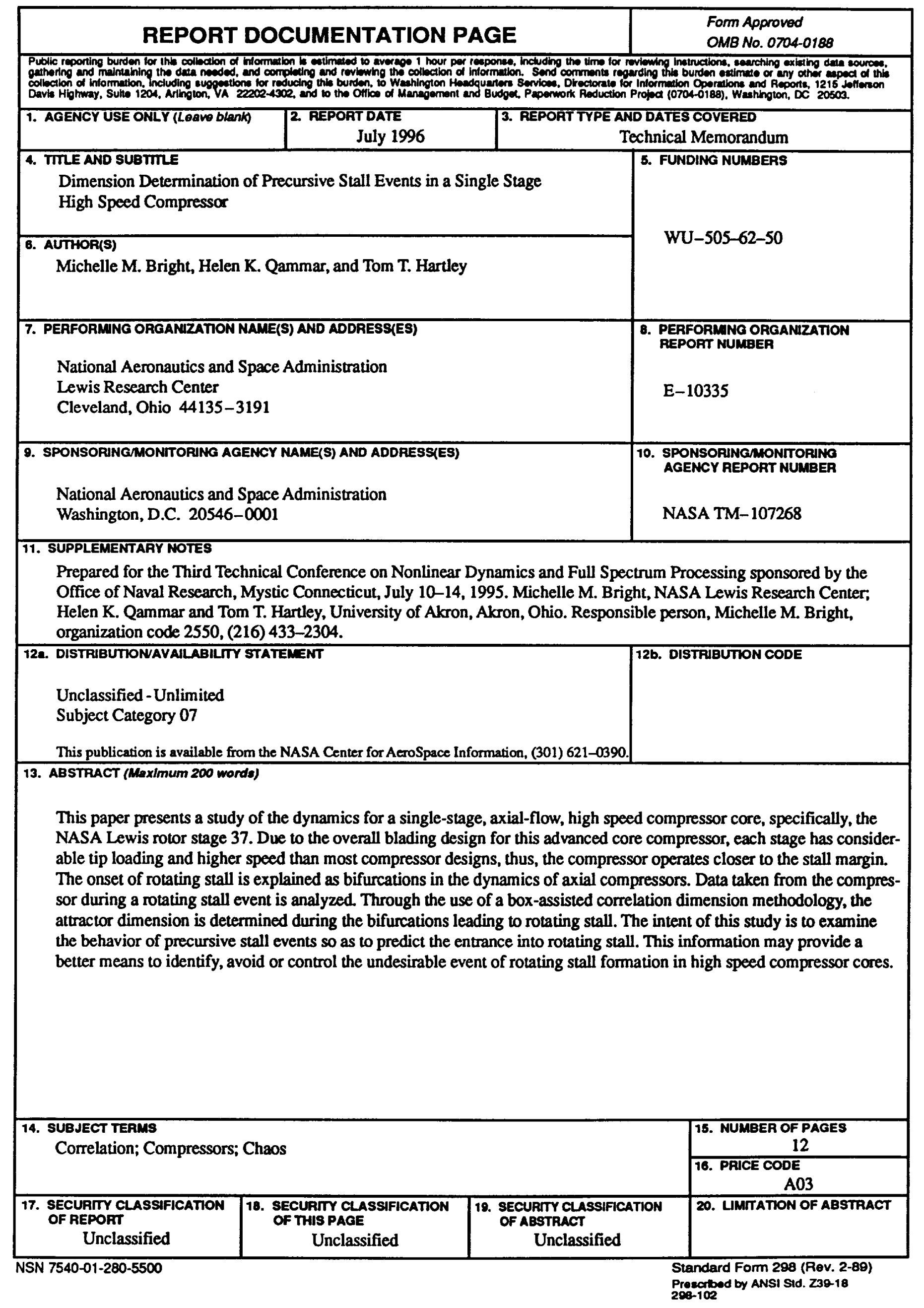

